\title{
Vaporization and pyrolysis modelling of a single droplet of heavy fuel oil using continuous thermodynamics
}

\author{
Vikrambhai Garaniya $^{1} \quad$ Laurie Goldsworthy ${ }^{2}$
}

(Received 3 August 2007; revised 11 October 2007)

\begin{abstract}
This article presents modelling of vaporization and pyrolysis of a single droplet of heavy fuel oil in a high ambient temperature field using the principle of continuous thermodynamics. Continuous thermodynamics reduces the computational simulation load compared with discrete thermodynamics, without compromising the quality of prediction of the complex combustion behaviours of such multicomponent complex fuels. Heavy fuel oil is represented by four fuel fractions and each of these fractions is assigned a separate distribution function. The prediction shows that in the modelling of heavy fuel oil, both heating rate and composition are very important parameters in coke formation. Coke formation can lead to engine degradation and increased exhaust smoke. The developed model shows good agreement with experimental results obtained by other researchers.
\end{abstract}

See http://anziamj.austms.org.au/ojs/index.php/ANZIAMJ/article/view/369 for this article, (C) Austral. Mathematical Soc. 2007. Published October 16, 2007. ISSN 1446-8735 


\section{Contents}

1 Introduction

C117

1.1 Heavy fuel oil . . . . . . . . . . . . . . . . . . . C C118

1.2 Distribution function . . . . . . . . . . . . . . . C119

1.3 Vapour phase . . . . . . . . . . . . . . . . . C122

1.4 Liquid phase . . . . . . . . . . . . . . . . . . . C125

1.5 Vapour liquid equilibrium . . . . . . . . . . . . . C C128

1.6 Energy equation-Droplet heating rate . . . . . . . . . C129

2 Results and discussion

C130

3 Conclusion

C135

A Notation

C135

References

C136

\section{Introduction}

The global shipping industry transports more than $85 \%$ of world trade and primarily uses heavy fuel oil (HFO) to power its engines. Successful design of ship engines for fuel efficiency and reduced emissions requires accurate modelling of the fuel. The present model describes vaporization and pyrolysis of a single droplet of HFO at high temperature and atmospheric pressure. The gas phase is considered as quasi-steady and its properties are time varying. The structural and composition chemistry of HFO is very complex, but the continuous thermodynamics technique uses the multi distribution function, which represents each fuel component with a simple Probably Density Function (PDF). This technique was originally developed by Tamim \& Hallett [1] for the fuel evaporation process. The continuous thermodynamics technique also has potential to describe the multicomponent fuel spray model which was 
confirmed by Lippert et al. [2, 3]. Recently, Hallett \& Clark [4] developed a continuous thermodynamics model of biomass pyrolysis oil evaporation and pyrolysis which shows good agreement with the experimental results.

\subsection{Heavy fuel oil}

Heavy fuel oil is produced by combining heavy residue from the crude oil refining process with lighter hydrocarbons, sometimes called cutter stock. The cutter stock is necessary to make the highly viscous residue useable as a fuel. Heavy fuel oil consists of a large number of hydrocarbons having huge molecular weight range and dissimilar structures [5], but the smallest molecules, primarily from the cutter stock, are either paraffins, naphthalenes or aromatics.

Chemical structure and molecular weight both are necessary to determine the behaviour of heavy fuel oil during heating and pyrolysis. A complete description of the chemical structure of molecules present in a heavy fuel oil is impossible due to the large range of molecular weight, but it can be described by different group contribution methods as mentioned by Baert [6].

When the molecular weight in $\mathrm{HFO}$ is below 500, the hydrocarbons are pure. Most hydrocarbons with molecular weight above 500 contain a small amount of $\mathrm{O}, \mathrm{N}$ and $\mathrm{S}$ (heteroatoms), which give a polar character to the compound. Aromaticity and polarity (number of heteroatoms) of the molecule increase with the increase in molecular weight. These polar molecules can be separated into two fractions, Asphaltene and Resin. With the increase in molecular weight, the complexity of hydrocarbons increases.

As shown in Table 1, HFO is assumed to contain four different chemical components: $n$-paraffins, naphthalene, aromatics and residue. Among these four, the properties of $n$-paraffins, aromatics and naphthalene are known, whereas the properties of the residue are not known experimentally. The residual portion of the heavy fuel oil may contain higher molecular weight 
TABLE 1: Distribution function parameters for the composition of $\mathrm{HFO}$

\begin{tabular}{lcccc}
\hline Components & Mass Frac & Dist Origin $(\gamma)$ & Dist mean $(\theta)$ & Std Devn $(\sigma)$ \\
\hline n-paraffins & 0.2 & 160 & 340 & 43.69 \\
Aromatics & 0.1 & 160 & 300 & 45.75 \\
Naphthalene & 0.2 & 160 & 370 & 45.47 \\
Residue & 0.5 & 500 & 850 & 320.15 \\
\hline
\end{tabular}

than used in the present study, because it mainly consists of asphaltene and aromatics. However, the very heavy molecules will not evaporate, but will thermally decompose into lighter compounds. The pyrolysis model accounts for the very heavy hydrocarbons.

The mean molecular weight and mass mean molecular weight used in the present study for heavy fuel oil are 488 and 597 respectively. In HFO, the residue is considered to have large hydrocarbons and also have some polar compounds, so it ignites very poorly but it produces coke and volatile gas thorough pyrolysis. The pyrolysis model requires aromaticity of the components that in this case has been taken as 0.4. Typical values of aromaticity of non cracked vacuum residue found for various hydrocarbons are $0.5,0.35$ and 0.3 for asphaltenes, resins and non-polar aromatic respectively.

Figure 1 shows the molecular weight distribution of the pure hydrocarbons, ranging from 160 to $500 \mathrm{~kg} / \mathrm{kmol}$. The second figure represents continuation of the molecular weight distribution beyond 500 to $1500 \mathrm{~kg} / \mathrm{kmol}$, representing the residue. The distribution parameters are chosen such they cover the range of molecular weight suggested by Baert [6].

\subsection{Distribution function}

The distribution function is any suitable function that describe the fluid mixture accurately. Here, the $\Gamma$-distribution function (Schultz or Pearson 


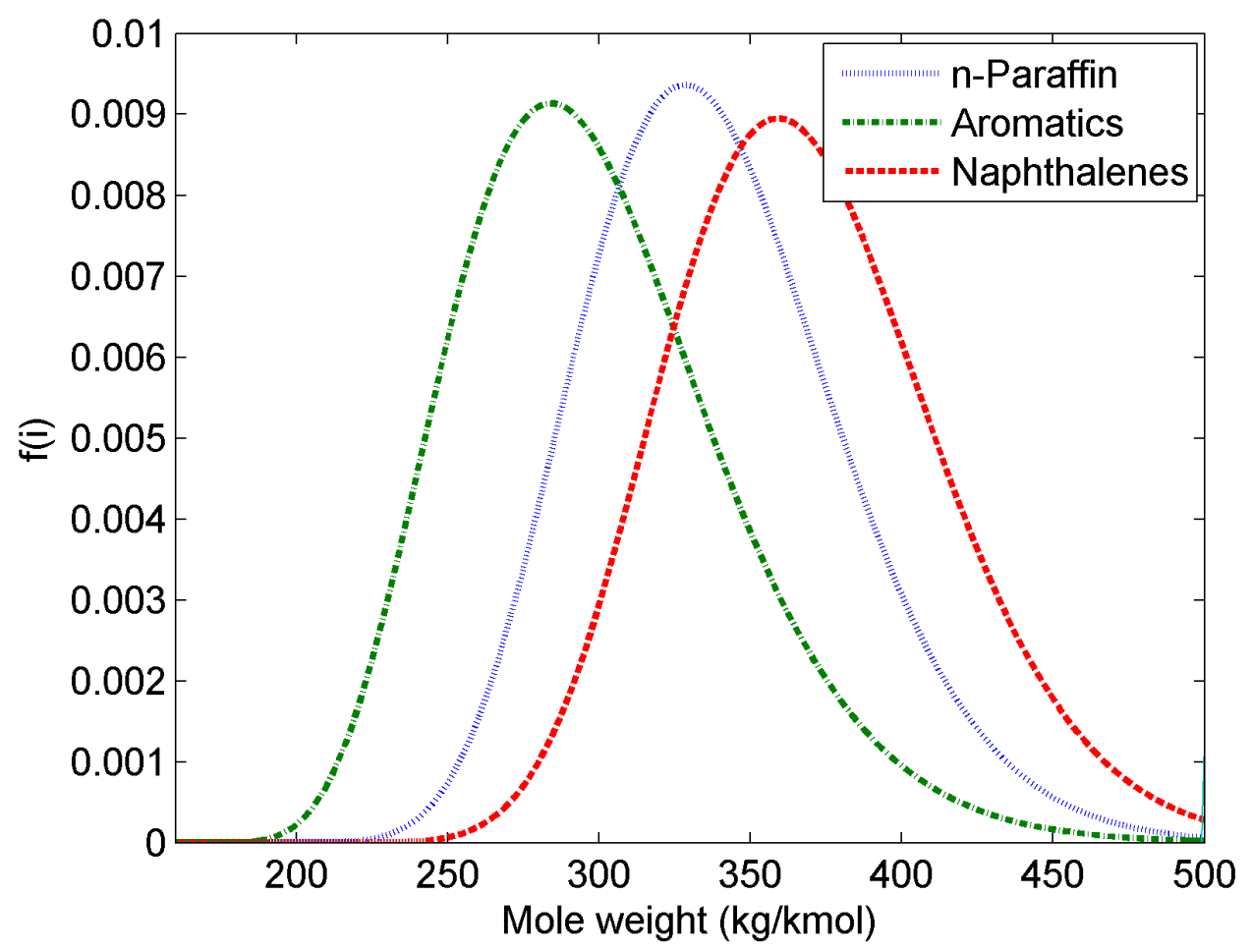

Figure 1: Distribution functions used for pure hydrocarbons of HFO. 


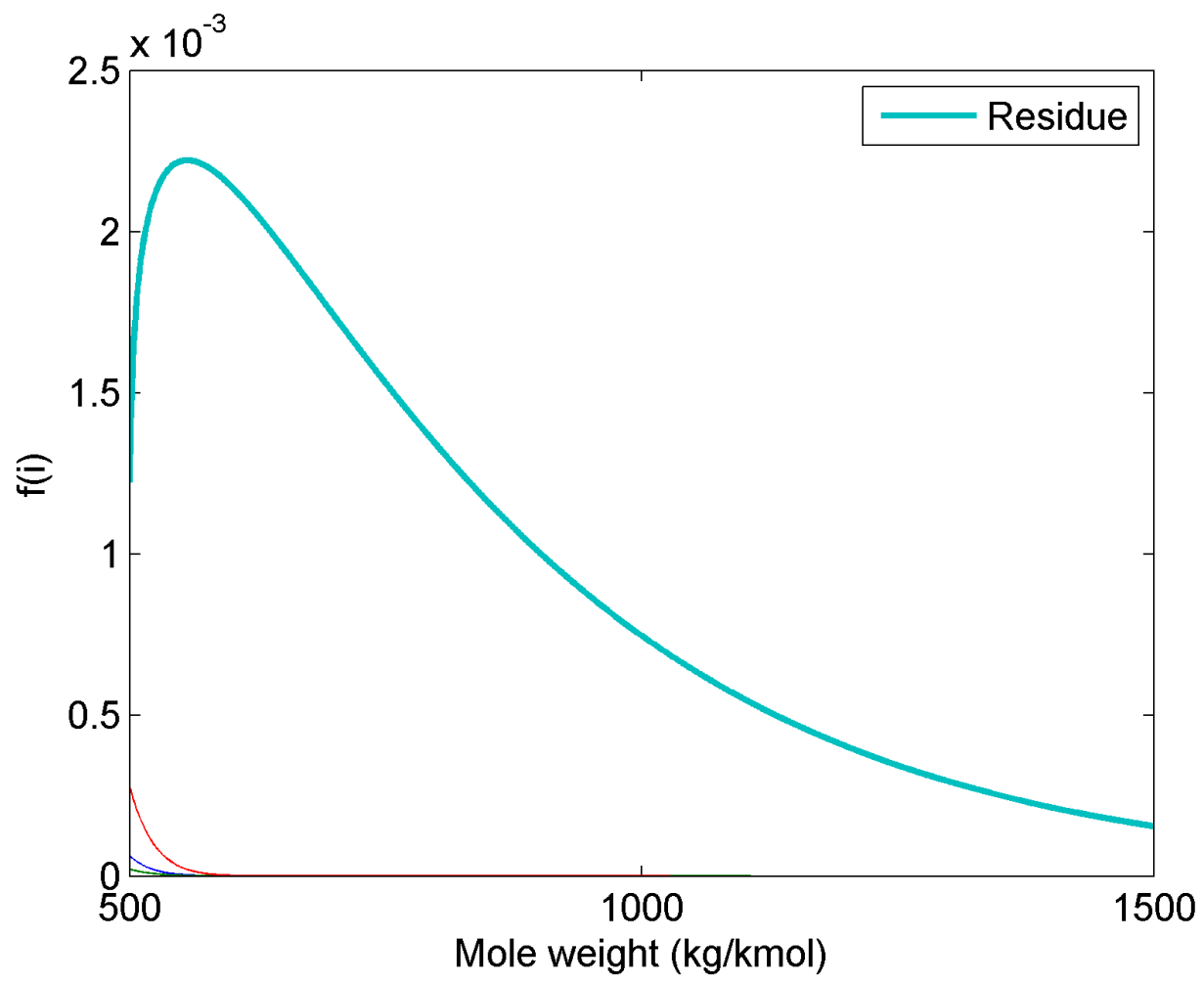

FiguRE 2: Distribution function used for residual portion of HFO. 
type III function) has been chosen. It was used by many researchers $[1$, $2,3,4,7,8]$ and has the ability to represent petroleum fractions. In the present modelling, the component's molecular weight has been chosen as the characterizing variable. The $\Gamma$-distribution is

$$
f_{j}(I)=\frac{\left(I-\gamma_{j}\right)^{\alpha_{j}-1}}{\beta_{j}^{\alpha_{j}} \Gamma\left(\alpha_{j}\right)} \exp \left[-\left(\frac{I-\gamma_{j}}{\beta_{j}}\right)\right] .
$$

The mean and variance of this function for the liquid phase are

$$
\theta_{L j}=\alpha_{L j} \beta_{L j}+\gamma_{L j} \quad \text { and } \quad \sigma_{L j}^{2}=\alpha_{L j} \beta_{L j}^{2} .
$$

\subsection{Vapour phase}

In this article, the liquid droplet contains $J$ hydrocarbon components with initial temperature as $T_{L 0}$ and it is abruptly exposed to surrounding temperature $T_{\infty}$. Here, each hydrocarbon component has been assigned with a separate distribution function $f_{L j}(I)$ along with two distribution parameters; mean $\theta_{L j}$ and variance $\sigma_{L j}^{2}$. In continuous thermodynamics modelling, these two distribution parameters are sufficient for accurate representation of the composition of each hydrocarbon. Vapour is produced by evaporation. Hence, the corresponding vapour phase distribution is $f_{V j}(I)$. The distribution variable $(I)$ can be any physical property, but here it represents the molecular weight of each hydrocarbon component. The molar concentrations of species $i$ of molecular weight $I$ are $f_{j}(I)$. Therefore, the vapour and liquid mole fraction of species $i$ are respectively given by Hallett \& Clark [4] as

$$
y_{i}=y_{F j} f_{V j}(I) d I \quad \text { and } \quad x_{i}=x_{F j} f_{L j}(I) d I,
$$

where $y_{F j}$ and $x_{F j}$ are the overall mole fraction of distribution $j$ in vapour and liquid phase respectively. The overall mole fraction of vapour phase $y_{F j}$, together with mole fraction of ambient air and pyrolysis volatile gases sum 
to unity, that is

$$
\sum_{j=1}^{J} y_{F j}+y_{A}+y_{G}=1 .
$$

Now, substituting $y_{i}$ from equation (3) into the species diffusion equation and integrating with $I^{n}$ (where $n=0,1,2$ ) as a weighting function and $d I$ as an interval as described by Hallett \& Clark [4], the transport equations of each species in vapour phase are derived as follows:

$$
\begin{aligned}
r^{2} c v^{*}\left(\frac{\partial y_{F j}}{\partial r}\right) & =c \bar{D}_{j} \frac{\partial}{\partial r}\left(r^{2} \frac{\partial y_{F j}}{\partial r}\right) \\
r^{2} c v^{*}\left(\frac{\partial\left(y_{F j} \theta_{j}\right)}{\partial r}\right) & =c \bar{D}_{j} \frac{\partial}{\partial r}\left(r^{2} \frac{\partial\left(y_{F j} \theta_{j}\right)}{\partial r}\right) \\
r^{2} c v^{*}\left(\frac{\partial\left(y_{F j} \psi_{j}\right)}{\partial r}\right) & =c \bar{D}_{j} \frac{\partial}{\partial r}\left(r^{2} \frac{\partial\left(y_{F j} \psi_{j}\right)}{\partial r}\right) .
\end{aligned}
$$

The above equations describe the transport of fuel vapour composition $y_{F j}$, distribution mean $\theta_{j}$ and second central moment of the distribution $\psi_{j}$. In these three equations, $c$ is molar density, $v^{*}$ is average species velocity, and $\bar{D}_{j}$ is average diffusivity. To avoid the complexity of modelling, molar densities and mole fractions are used in the above equations throughout instead of mass. There are three $\bar{D}_{j}$ arising from the above equations as mentioned by Tamim \& Hallett [1], but their values are almost indistinguishable [8]. Therefore, they are assumed identical. The molar flux leaving the droplet surface with molar average velocity is represented by Hallett \& Clark [4] as

$$
c v^{*} r^{2}=N R^{2}
$$

Spherical symmetry has been assumed here and the boundary condition at the droplet surface and ambient is

- At the droplet surface $(r=R) ; y_{F j}=y_{F j R} ; y_{F j} \theta=y_{F j R} \theta_{R} ; y_{F j} \psi=$ $y_{F j R} \psi_{R}$ and $T=T_{R}$ 
- At ambient $(r=\infty) ; y_{F j}=y_{F j \infty} ; y_{F j} \theta=y_{F j \infty} \theta_{\infty} ; y_{F j} \psi=y_{F j \infty} \psi_{\infty}$ and $T=T_{\infty}$

- It is assumed that $y_{F j \infty}=0$; hence, $\left(y_{F j} \theta\right)_{\infty}=\left(y_{F j} \psi\right)_{\infty}=0$.

Here it is postulated that, from the beginning the residue in the liquid phase contains high molecular weight and during evaporation it evaporates very little, but it produces volatile gas and coke due to pyrolysis. Therefore, the total molar flux $N$ should include this volatile gas along with the fuel vapour:

$$
\xi_{j}=N_{j} / N \quad \text { and } \quad \xi_{G}=N_{G} / N .
$$

The total vaporizing molar flux of the droplet is

$$
N=\sum_{j=1}^{J} N_{j}+N_{G} .
$$

The solution of equation (5) and (8) is [7]

$$
N=\frac{c \bar{D}_{j}}{R} \ln \left(1+B_{j}\right), \quad \text { where } \quad B_{j}=\frac{y_{F j R}-y_{F j \infty}}{\xi_{j}-y_{F j R}} .
$$

The above theory can be extended to include the effect of convection using Sherwood number Sho at low mass transfer rates [4] as

$$
N=\operatorname{Sho} \frac{c \bar{D}_{j}}{2 R} \ln \left(1+B_{j}\right) \text {. }
$$

The $\xi_{j}$ 's used in above equation may be found by equating equation (12) for $j=1$ and $j=j$,:

$$
\xi_{j}=y_{F j R}+\frac{y_{F j R}-y_{F j \infty}}{\exp \left(2 N R / c \bar{D}_{j} \text { Sho }\right)-1} .
$$

Here, equation (12) and (13) gives a set of simultaneous equation which must be solved simultaneously to calculate the $\xi_{j}, N$ and $N_{G}$. Now, equation (5), 
(6) and (7) can be solved for the variation of $y_{F j}, y_{F j} \theta_{j}$ and $y_{F j} \psi_{j}$ in space (beyond the droplet surface) as

$$
\begin{aligned}
& y_{F j}(r)=\xi_{j}-\left(\xi_{j}-y_{F j \infty}\right)\left(1+B_{j}\right)^{-Z} \\
& y_{F j} \theta_{j}(r)=y_{F j R} \theta_{j R}-\frac{1}{B_{j}}\left(y_{F j R} \theta_{j R}-y_{F j \infty} \theta_{j \infty}\right)\left[\left(1+B_{j}\right)^{(1-Z)}-1\right], \\
& y_{F j} \psi_{j}(r)=y_{F j R} \psi_{j R}-\frac{1}{B_{j}}\left(y_{F j R} \psi_{j R}-y_{F j \infty} \psi_{j \infty}\right)\left[\left(1+B_{j}\right)^{(1-Z)}-1\right],(16) \\
& \text { where } \quad Z=1+\frac{\text { Sho }}{2}\left[\left(\frac{R}{r}\right)-1\right] .
\end{aligned}
$$

The present model requires transport properties of both the liquid and vapour phases. These transport properties are usually dependent on temperature and concentration of the mixture. Therefore, these must be evaluated at some reference temperature and concentration. Vapour phase properties are calculated using the one third rule of reference state as described by Hallett [8].

\subsection{Liquid phase}

This article assumes that mixing in the liquid phase is rapid enough to give uniform temperature and composition throughout the evaporation and pyrolysis processes. The liquid phase undergoes the following processes: 1) Preignition heating, 2) Evaporation, 3) Thermal decomposition, and 4) Polymerization.

Generally, droplets rise to temperature around $700 \mathrm{~K}$ during the evaporation stage [9]. Pyrolysis is a process in which chemical changes of organic compounds are caused by heating in the absence of oxygen. At sufficiently high temperature, the molecules start decomposing into radicals. These radicals can either decompose further or recombine through polymerization. 
Therefore, these reactions convert the higher molecules into low molecular weight gases and high molecular weight coke residue [6]. The following pyrolysis model is used in the present study.

1. Initially the mass of the fuel component is $\left(m_{L j}\right)$, which converts to volatile gas $\left(m_{G j}\right)$ through cracking and into polymer through polymerization $\left(m_{P j}\right)$.

2. Residue is initially present in the fuel.

3. Most of the pure hydrocarbons evaporate before thermal cracking occurs.

4. The volatile products of cracking diffuse rapidly to the ambient atmosphere.

5. The tendency to polymerization increases with aromaticity.

In the liquid phase, due to thermal cracking the molecular weight of fuel decreases, but only the non-aromatic part of the molecules produces volatiles through cracking. This decomposition rate exponentially depends on temperature [6].

Generally, heavy fuel oil polymerization starts at $840 \mathrm{~K}$. Thermal decomposition also takes place simultaneously with polymerization [10]. When heavy fuel oil completely burns, it leaves the droplet with the skeleton of the carbonaceous material, which is called a cenosphere. In the liquid phase, polymerization results in a decrease of the number of monomers from the fuel, but only the aromatic part of the monomers combines and produces polymers. This polymerization rate depends exponentially on temperature and is independent of molecular weight [6]. During the liquid phase pyrolysis, polymer formation occurs continuously, but at the same time the non-aromatic part of the polymer undergoes further cracking due to high liquid temperature. Moreover, this polymerization rate shows dependence 
on temperature and aromaticity $(A R)$. Hence, the combined rate of volatile gas production by thermal cracking of liquid and cracking of the polymer for a single fraction is given by Baert [6] as

$$
\frac{d m_{G j}}{d t}=\left(m_{L j}+m_{P j}\right)\left[k_{1} e^{-E_{1} / \mathcal{R} T}\left(1-A R_{j}\right)\right] .
$$

The rate of polymer formation through polymerization of a single fraction of the liquid is

$$
\frac{d m_{P j}}{d t}=m_{P j}\left[k_{1} e^{-E_{1} / \mathcal{R} T}\left(1-A R_{j}\right)\right]+m_{L j}\left[\left(k_{2} e^{-E_{2} / \mathcal{R} T}+k_{3} e^{-E_{3} / \mathcal{R} T}\right) A R_{j}\right],
$$

where all Arrhenius law parameters are the same as those used by Baert [6].

A molar flux balance on the surface gives the change of composition of the liquid phase during evaporation and pyrolysis. In this modelling, it has been assumed that all components of the residual portion pyrolise at the same rate, so no molecular weight change occurs due to pyrolysis. Hence, following Hallett et al. [4, 7], the change in mean molecular weight of liquid through evaporation and pyrolysis in terms of continuous thermodynamics is

$$
\frac{d \theta_{L j}}{d t}=\frac{3 N}{x_{j} c_{L} R}\left[\theta_{L j} \xi_{j}+\frac{\theta_{j \infty} y_{F j \infty}-\theta_{j R} y_{F j R}\left(1+B_{j}\right)}{B_{j}}\right] .
$$

An analogous equation is obtained for $\frac{d \psi}{d t}$ as

$$
\frac{d \psi_{L j}}{d t}=\frac{3 N}{x_{j} c_{L} R}\left[\psi_{L j} \xi_{j}+\frac{\psi_{j \infty} y_{F j \infty}-\psi_{j R} y_{F j R}\left(1+B_{j}\right)}{B_{j}}\right] .
$$

Hence, the mass of a single fraction at any time is

$$
m_{L j}=m_{L j(t=0)}-m_{V j}-m_{G j}-m_{P j} .
$$




\subsection{Vapour liquid equilibrium}

To calculate the rate of diffusion of vapour to the ambient gas, it is first necessary to find the mole fraction of each vapourised component at the droplet surface. In the present study, the liquid is assumed an ideal solution whose vapour phase can be approximated by an ideal gas mixture. The VLE can be expressed in terms of continuous thermodynamics and Raoult's law as

$$
y_{F j R}=x_{F j R} \int_{0}^{\infty} f_{L j}(I) \frac{P_{v}(I)}{P} d I .
$$

The Clausius-Clapeyron equation is used to determine the vapour pressure of a component at the droplet surface [1] as

$$
P_{v}(I)=P_{A T M} \exp \left[\left(\frac{S_{f g}}{\mathcal{R} T}\right)\left(T-T_{b}(I)\right)\right],
$$

where $S_{f g}$ is the entropy of vaporization and $T_{b}(I)$ the component's boiling point at atmospheric pressure. Now, substituting the distribution function into the above equation and integrating with $I^{n}$ as a weighting factor, vapour phase mole fraction and other distribution parameters at the droplet surface are obtained for a single component $[1,2,8]$ are

$$
\begin{aligned}
& y_{F j R}=\frac{P_{A T M} \exp \left[\left(\frac{S_{f g j}}{\mathcal{R} T_{R}}\right)\left(T_{R}-a_{B}-\gamma_{j} b_{B}\right)\right]}{P_{\infty}\left[1+\left(\frac{S_{f g j}}{\mathcal{R} T_{R}}\right) b_{B} \beta_{L j}\right]^{\alpha_{L j}}}, \\
& \theta_{R j}=\gamma_{j}+\frac{\theta_{L j}-\gamma_{j}}{1+\left[\left(\frac{S_{f g j} b_{B}}{\mathcal{R} T_{R}}\right) \frac{\sigma_{L j}^{2}}{\left(\theta_{L j}-\gamma_{j}\right)}\right]}, \\
& \sigma_{R j}^{2}=\sigma_{L j}^{2}\left(\frac{\theta_{R j}-\gamma_{j}}{\theta_{L j}-\gamma_{j}}\right)^{2} .
\end{aligned}
$$




\subsection{Energy equation-Droplet heating rate}

The energy balance for a droplet of continuous mixture is given by assuming quasi-steady vaporization [8] as

$$
c v^{*} C_{p V} \frac{\partial T}{\partial r}=\frac{\lambda}{r^{2}} \frac{\partial}{\partial r}\left(r^{2} \frac{\partial T}{\partial r}\right) .
$$

The vapour phase energy equation is solved to give heat transfer to the droplet as

$q=\left(\frac{N u o \lambda \ln \left(1+B_{T H}\right)}{2 R B_{T H}}\right)\left(T_{\infty}-T_{R}\right), \quad$ where $\quad B_{T H}=\exp \left(\frac{2 N C_{p V}}{\lambda N u o}\right)-1$.

In the above equation, $C_{p V}$ is the specific heat of the vapour phase, which only includes fuel vapour and pyrolysis volatile gas. Hence, the droplet heating rate can be given for uniform liquid temperature as

$$
\frac{d T_{R}}{d t}=\frac{3}{C_{p L} c_{L} R}\left(q-N h_{f g}\right)
$$

Here, $q$ is the conduction heat flux. Radiation heat transfer is neglected. By substituting $\rho_{L}=c_{L} \theta_{L}$, the equation (30) becomes

$$
\frac{d T_{R}}{d t}=\frac{\theta_{L} A}{m_{L} C_{p L}}\left(q-N h_{f g}\right)
$$

Now, considering the decomposition enthalpy of the pyrolysis and including the specific heat of coke, the change in droplet temperature $\left(T_{R}\right)$ with time is

$$
\frac{d T_{R}}{d t}=\frac{\theta_{L} A}{\left(m_{L} C_{p L}+m_{P} C_{p P}\right)}\left(q-N h_{f g}-N_{G} h_{\mathrm{dec}}\right) .
$$




\section{Results and discussion}

To illustrate the model, sample calculations are presented here for a single droplet of HFO of 100 micron diameter. The initial temperature of a droplet is $300 \mathrm{~K}$ and it is exposed to surrounding temperature $1200 \mathrm{~K}$. The molecular weight history of the pure hydrocarbon components of a single HFO droplet is given in Figure 3. As the liquid droplet enters the hot ambient surrounding it is heated and starts vaporizing into the surroundings. This evaporation favours the fractional distillation of the light and more volatile hydrocarbons. As a result of this, the mean molecular weight $\left(\theta_{L j}\right)$ of the pure hydrocarbons increases and the standard deviation $\left(\sigma_{L j}\right)$ decreases. This concurs with experimental results given by Ikegami et al. [10], which show preferential distillation like evaporation of the light components of a HFO droplet.

Figure 4 shows the history of the liquid temperature $\left(T_{R}\right)$. The temperature of the liquid steadily increases with droplet lifetime. The droplet temperature does not reach a steady value as would be expected with a pure compound. The temperature goes on increasing because of heat transfer and because the effective boiling temperature increases due to fractional distillation. Once evaporation finishes, the droplet mass loss is due to evolution of gas from thermal decomposition. The rate of mass loss by this mechanism is initially less than that by evaporation, and the energy absorbed by the endothermic thermal decomposition is less than the evaporation enthalpy of the evaporating liquid. In the present model, enthalpy of decomposition is included in the calculation of droplet energy. The predicted liquid temperature history coincides with observations of Shyu et al. [11].

Figure 5 shows scaled droplet diameter squared with time. During the initial period of the droplet's lifetime, the square of a droplet diameter decreases very rapidly due to preferential evaporation of the most volatile components. As the light components deplete from the liquid, the overall rate of vaporization slows. As the droplet temperature continues to increase beyond about 0.04 seconds, pyrolysis of the liquid, producing gas, becomes significant. The 


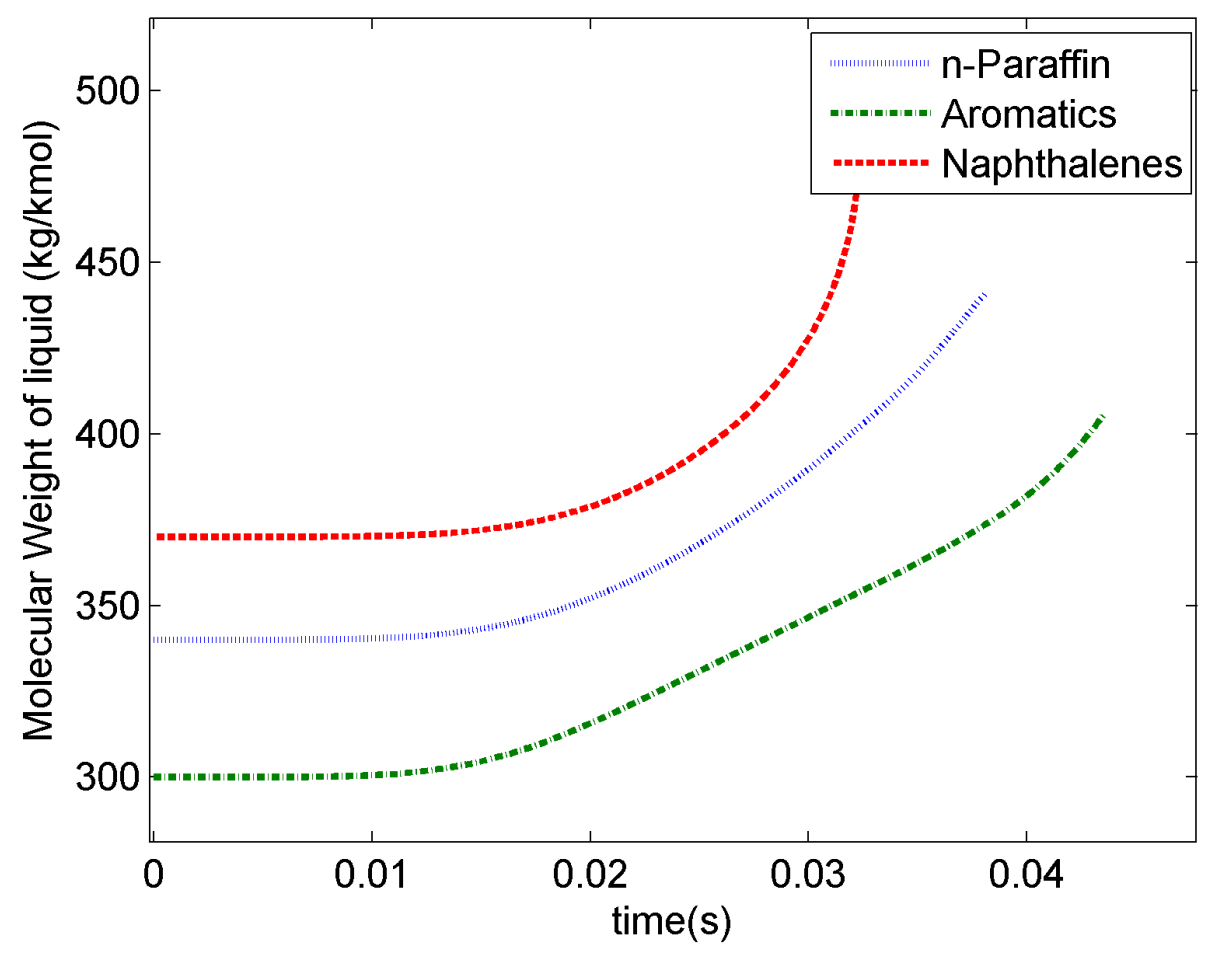

FiguRE 3: Predicted composition history of 100 micron HFO droplet. 


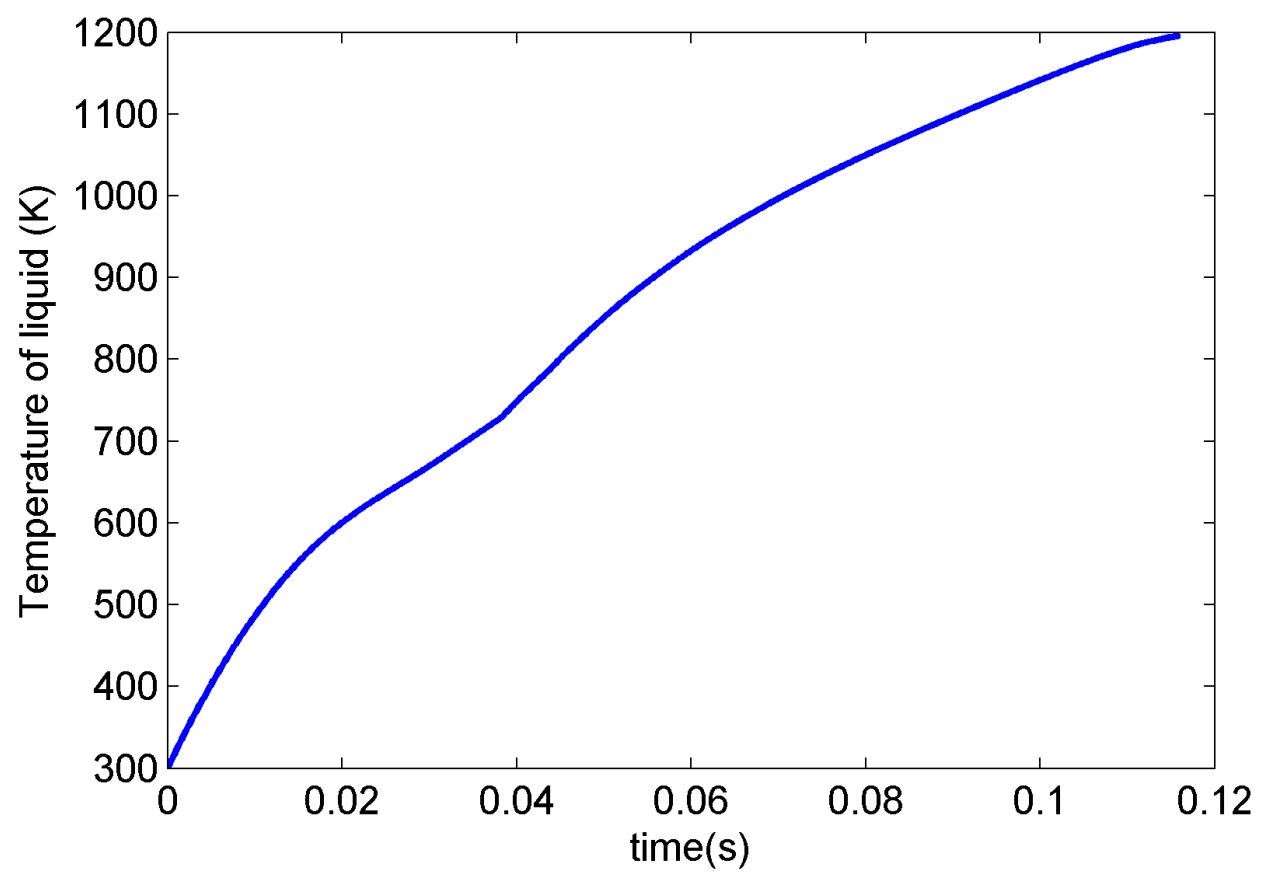

Figure 4: Predicted liquid temperature history of 100 micron HFO droplet. 


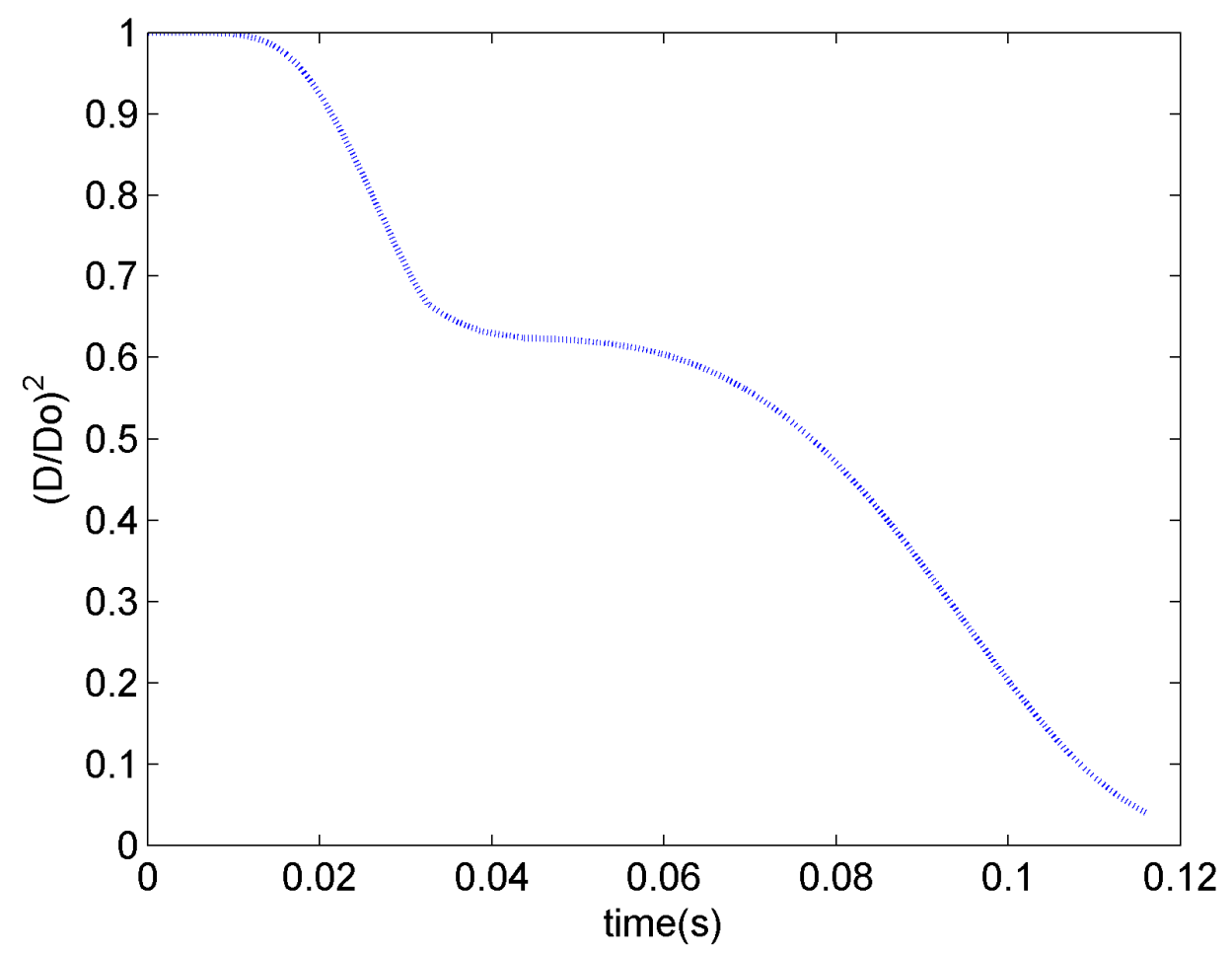

FiguRE 5: Predicted droplet size history of 100 micron HFO droplet. 


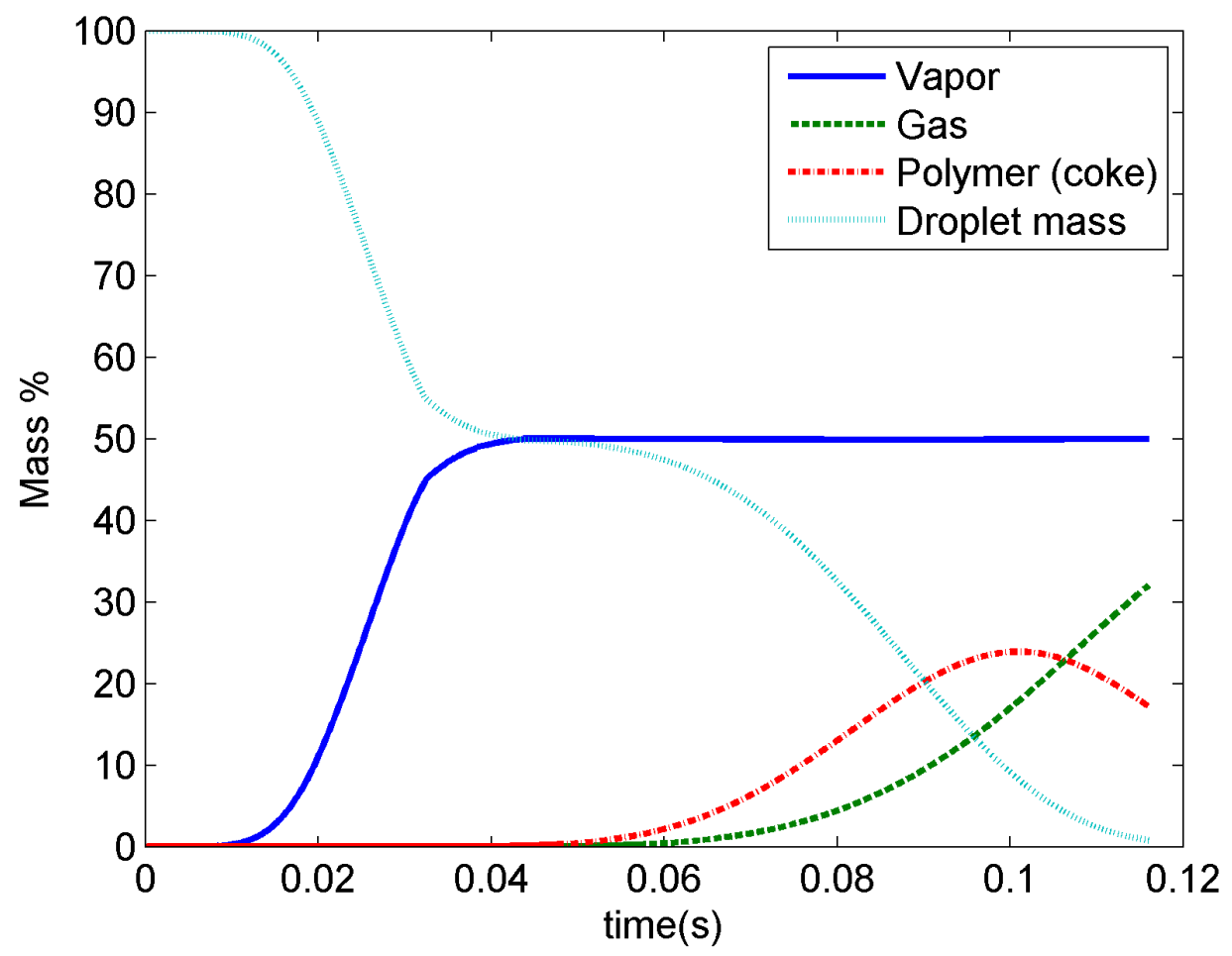

Figure 6: Predicted mass history of 100 micron HFO droplet.

straight line $d^{2}$ law typical of single component liquids is not reproduced for a heavy fuel oil droplet. Here, Figure 6 shows the mass of vapour from evaporation, the mass of polymer and the mass of pyrolysis gas. Vapour is produced only from the pure hydrocarbons. The gas and coke are only formed from the residual portion. The amount of polymer at the end of the droplet lifetime is less than the maximum during pyrolysis. The reason for this is that formed polymer also decomposes at high temperature due to thermal cracking and produces volatile gas. 


\section{Conclusion}

The model presented calculates the vaporisation and pyrolysis process of heavy fuel oil using continuous thermodynamics. In multicomponent fuel modelling, composition is simply represented by two parameters for each of a number of classes of components, instead of thousands of property values and mole fractions of the individual components, as required in conventional discrete modelling methods. The present model demonstrates that continuous thermodynamics combined with chemical kinetics has the ability to model the evaporation and pyrolysis processes for such complex mixtures.

Acknowledgements: We acknowledge the important discussions with Prof William Hallett from University of Ottawa and Luciano Mason here at Australian Maritime College.

\section{A Notation}

$A$ Surface area of the droplet, $\mathrm{m}^{2}$

$E \quad$ Activation energy, $\mathrm{kJ} / \mathrm{kmol}$

$R$ Radius of the droplet, $\mathrm{m}$

$\mathcal{R}$ Universal gas constant, $\mathrm{kJ} / \mathrm{kmol} \mathrm{K}$

$V$ Droplet volume, $\mathrm{m}^{3}$

$r$ Radial coordinate beyond droplet surface, $\mathrm{m}$

$t$ Time, s

$x \quad$ Liquid phase mole fraction

$y \quad$ Vapour phase mole fraction

$\lambda$ Thermal conductivity, $\mathrm{W} / \mathrm{m} \mathrm{K}$

$\Phi_{D}$ Temperature dependence

\section{Subscripts}

A Air 

$F \quad$ Fuel
$L \quad$ Liquid
$G$ Pyrolysis Gas
$P$ Polymer
$V$ Vapour phase
$j \quad$ Index for a fuel fraction (1 to 4 )

\section{References}

[1] Tamim, J. and W. L. H. Hallett, A continuous thermodynamics model for multicomponent droplet vaporization. Chemical Engineering Science, 1995, 50(18): pp. 2933-2942. doi:10.1016/0009-2509(95)00131-N C117, C122, C123, C128

[2] Lippert, A. M. and R. D. Reitz. Modelling of multicomponent fuels using continuous distributions with application to droplet evaporation and sprays. in International Fall Fuels and Lubricants Meeting and Explosion. 1997. Tulsa, Oklahoma, 972882. C118, C122, C128

[3] Lippert, A. M., D. W. Stanton, C. J. Rutland, W. L. H. Hallett, and R. D. Reitz, Multidimensional simulation of diesel engine cold start with advanced physical submodels. International Journal of Engine Research, 2000, 1(1): pp. 1-27. C118, C122

[4] Hallett, W. L. H. and N. A. Clark, A model for the evaporation of biomass pyrolysis oil droplets. Fuel, 2006, 85(4): pp. 532-544. doi:10.1016/j.fuel.2005.08.006 C118, C122, C123, C124, C127

[5] Goldsworthy, L., CFD Modelling of residual fuel oil combustion in the context of marine diesel engines. International Journal of Engine Research, 2005, 7: pp. 181-199. C118 
[6] Baert, R. S. G., A mathematical model for heavy fuel oil droplet vaporization and pyrolysis in a heavy temperature inert gas. Combustion Science and Technology, 1993, 90: pp. 125-147. C118, C119, C126, C127

[7] Hallett, W. L. H and C. Grimwood. A simple continuous mixture droplet evaporation model with multiple distribution functions. in Combustion Institute, 2001. Canadian Section. Spring Meeting. C122, C124, C127

[8] Hallett, W. L. H., A simple model for the vaporization of droplets with large numbers of components. Combustion and Flame, 2000, 121(1-2): pp. 334-344. doi:10.1016/S0010-2180(99)00144-3 C122, C123, C125, C128, C129

[9] Chen, C. S. and M. M.EI-Wakil, Experimental and theoretical studies of burning drops of hydrocarbon mixtures. Proceedings for Instrumentation and Mechanical Engineers, 1969, 184 (Pt. 3J): pp. 95-108. C125

[10] Ikegami, M., G. Xu, K. Ikeda, S. Honma, H. Nagaishi, D. L. Dietrich, and Y. Takeshita, Distinctive combustion stages of single heavy oil droplet under microgravity. Fuel, 2003, 82(3): pp. 293-304. doi:10.1016/S0016-2361(02)00257-0 C126, C130

[11] Shyu, R. R., C. S. Chen, G. O. Goudie, and M. M. El-Wakil, Multi-component heavy fuel drop histories in a high-temperature flow field. Fuel, 1972, 51(2): pp. 135-145. C130 


\section{Author addresses}

1. Vikrambhai Garaniya, Dept of Maritime Engineering, Australian Maritime College, Launceston, Tasmania 7250, Australia. mailto:v.garaniya@amc.edu.au

2. Laurie Goldsworthy, Dept of Maritime Engineering, Australian Maritime College, Launceston, Tasmania 7250, Australia. 